\title{
Burnout among Physicians and Nurses during COVID-19 Pandemic
}

\author{
Sezer Kisa ${ }^{1 *}$
}

${ }^{1}$ Oslo Metropolitan University, Faculty of Health Sciences, Department of Nursing and Health Promotion, 0166 Oslo, NORWAY

*Corresponding Author: sezerkisa@hotmail.com

Citation: Kisa, S. (2020). Burnout among Physicians and Nurses during COVID-19 Pandemic. European Journal of Environment and Public Health, 4(2), em0055. https://doi.org/10.29333/ejeph/8447

\begin{tabular}{ll}
\hline ARTICLE INFO & ABSTRACT \\
\cline { 1 - 1 } Received: 6 Jun. 2020 & $\begin{array}{l}\text { Health professionals treating COVID-19 patients have been reported to suffer extreme stress, which can lead to } \\
\text { burnout and a reduced capacity to continue work. Physicians and nurses are directly at risk when performing the } \\
\text { treatment and care of COVID-19 patients and therefore experience intense stress. This paper highlights the } \\
\text { individual, organizational, and social effects of burnout among physicians and nurses due to COVID-19. } \\
\text { Moreover, evidence based interventions were discussed to promote a healthy workplace, and to prevent burnout } \\
\text { among health professionals during the COVID-19 pandemic. }\end{array}$
\end{tabular}

Keywords: burnout, COVID-19, physicians, nurses

\section{INTRODUCTION}

COVID-19 disease, which emerged in Wuhan, China in December 2019, had quickly spread throughout the world (Hu et al., 2020). The spread of COVID-19 had developed more quickly than expected and was not expected to flow the epidemiological information necessary for the control of the disease and the creation of the surveillance system. In addition, in response to the increasing number of patients and workload in health institutions, many countries faced the problem of insufficient number of health personnel (Wu et al., 2020). Some country leaders called on retired doctors and nurses to support the fight against the COVID-19 outbreak, and many of the retired healthcare professionals responded positively to this call and joined the fight against the virus in their country (Iserson, 2020). In some countries, within the scope of combating the epidemic of COVID-19, regarding sustainability of health services, it was given importance to the continuity of the employment of health personnel in health facilities, and the health personnel working in health institutions by official authorities were not allowed to leave health care institutions (Anadolu Ajansi, 2020).

Health professionals often feel inadequate and unprotected against COVID-19. Reasons that reveal this sense of inadequacy and vulnerability included the insufficiency of scientific information about COVID-19, the belief that the vaccine / drug that will cure the disease cannot be discovered, the problems experienced in the provision of protective materials, the risk of healthcare professionals getting infected, and the healthcare personnel's risk of transmitting the disease (Greenberg et al., 2020; Wu et al., 2020). The perception of health personnel as a threat by the others to carry and transmit the disease to others have reached such a high level that some health professionals were forced to live isolated by their neighbors and relatives. Although the administration of many countries created interventions such as the supply of disposable materials, the establishment of housing facilities for health personnel, the support of the community, and psychological assistance, it has not solved the problem of burnout experienced by health professionals. Health professionals treating COVID-19 patients have been reported to suffer extreme stress, which can lead to burnout and a reduced capacity to continue work. One postulated reason for this is the 'moral injuries' they endure when, for example, deciding which patient with COVID-19 should receive a lifesaving therapy that is in short supply, at the expense of another dying patient (Greenberg et al., 2020). The problem of burnout continues to be an important risk factor affecting the quality of life and health of important health professionals with the rise of the pandemic.

\section{FACTORS CONTRIBUTING TO PHYSICIAN AND NURSE BURNOUT}

It is mainly physicians and nurses who have the most contact with patients and spend time with them among health professionals. Physicians and nurses are directly at risk when performing the treatment and care of COVID-19 patients and therefore experience intense stress. They are affected negatively by physical, mental, and social issues due to the problems they experience in the working environment and 
face the problem of burnout (Talee et al., 2020). Although physicians and nurses who experience burnout begin to realize the stressful work environments, the intensity of their workloads, and their own health problems caused by COVID19, they are unable to express these feelings due to the intensity of their work tempos, and become tired of their jobs over time (Hu et al., 2020).

The individual, organizational, and social effects of burnout among physicians and nurses due to COVID-19 have become clear. Symptoms of burnout in the way of psychophysiological, psychological, and behavioral were observed in physicians and nurses on the individual level. The most commonly reported psycho-physiological symptoms were tiredness, gastro-intestinal disorders and weight loss, coronary heart disease, breathing difficulties, and general pains (Wu et al., 2020). Psychological symptoms included emotional exhaustion, quick temper, being suspicious and anxious, irritability, helplessness, easy crying, decreased selfesteem and self-confidence, difficulty in concentration, apathy towards patients and depression. Reported behavioral burnout symptoms were physical and psychological distancing from patients, making similar mistakes, postponing work, deterioration in the nature of the service offered, deterioration in relationships at work and outside work, decrease in job performance, job dissatisfaction, conflict with family members and friends, increase use of drug, alcohol, and smoking (Hu et al., 2020: Talaee et al., 2020; Wu et al., 2020). Symptoms reported on the organizational level included a decrease in health service performance, negative job attitudes, an increase in the number of staff considering quitting, and increased service delivery costs (Hoffmeyer et al., 2020).

\section{INTERVENTIONS}

Burnout caused by COVID-19 is preventable and the most effective response and prevention strategy is early detection of these symptoms. To solve the problem, a greater focus needs to be placed on the individual and organizational factors that reveal burnout seen primarily among physicians and nurses. While focusing on individual factors, it should be aimed at increasing commitment to work by creating the best harmony between work and individual, and highlighting the sanctity of the profession. Intervention strategies for organizational factors should focus on social support, increasing participation in decision-making processes and improving communication with the administration (Greenberg et al., 2020). Nine evidence-based organizational strategies are suggested for health system leaders and managers to promote engagement and reduce burnouts: acknowledging and assessing the problem, harnessing the power of effective leadership, developing and implementing targeted interventions, cultivating community at work, using rewards and incentives wisely, aligning values and strengthen culture, promoting flexibility and work-life integration, providing resources to promote resilience and self-care, and facilitating and funding organizational science (Hofmeyer et al., 2020).

For future outbreaks similar to COVID-19, protective and supportive measures should be taken to protect the mental health of all health professionals in addition to measures to protect their physical health. To reduce the effects of COVID19 in many countries, counseling, and psychotherapy services were started to be provided to health professionals by establishing counseling teams including their psychiatrists. In some countries, peer support programs, regular meeting times, and psychiatric tele-consultation helplines have been established to address the mental health problems of health professionals facing overwork, stress, difficult ethical decisions, and multiple deaths (Greenberg et al., 2020).

\section{CONCLUSION}

The physical and mental health of all health care professionals is important for the sustainable delivery of health care in the fight against COVID-19. For this reason, it is important to identify the factors that may cause stress and burnout in health professionals and to develop institutional and national strategies and policies to eliminate these factors. Evidence based interventions should be also adopted and developed to promote a healthy workplace, addressing ethical issues, and preventing burnout among health professionals during the COVID-19 pandemic.

\section{REFERENCES}

Anadolu Ajansi. (2020). Turkey's health workers not allowed to leave jobs. (n.d.). https://www.aa.com.tr/en/latest-oncoronavirus-outbreak/turkey-s-health-workers-notallowed-to-leave-jobs/1782673

Greenberg, N., Docherty, M., Gnanapragasam, S., \& Wessely, S. (2020). Managing mental health challenges faced by healthcare workers during COVID-19 pandemic. BMJ, m1211. https://doi.org/10.1136/bmj.m1211

Hofmeyer, A., Taylor, R. and Kennedy, K. (2020). Fostering compassion and reducing burnout: How can health system leaders respond in the COVID-19 pandemic and beyond? Nurse Education Today, 104502. https://doi.org/10.1016/j.nedt.2020.104502

Hu, D., Kong, Y., Li, W., Han, Q., Zhang, X., Zhu, L. X., et al. (2020). Frontline nurses' burnout, anxiety, depression, and fear statuses and their associated factors during the COVID-19 outbreak in Wuhan, China: A large-scale crosssectional study. EClinicalMedicine, 100424. https://doi.org/10.1016/j.eclinm.2020.100424

Iserson, K. (2020). Augmenting the disaster healthcare workforce. WestJEM 21.3 May Issue, 21(3), 490-496. https://doi.org/10.5811/westjem.2020.4.47553

Talaee, N., Varahram, M., Jamaati, H., Salimi, A., Attarchi, M., Kazempour dizaji, M., Sadr, M., et al. (2020). Stress and burnout in health care workers during COVID-19 pandemic: Validation of a questionnaire. Journal of Public Health. Published online: 6 June 2020. https://doi.org/10.1007/s10389-020-01313-z 
Wu, Y., Wang, J., Luo, C., Hu, S., Lin, X., Anderson, A. E., Bruera, E., Yang, X., Wei, S. and Qian, Y. (2020). A comparison of burnout frequency among oncology physicians and nurses working on the frontline and usual wards during the COVID-19 epidemic in Wuhan, China. Journal of Pain and Symptom Management, 60(1), e60-e65. https://doi.org/10.1016/j.jpainsymman.2020.04.008 